LA GRANJA:

REVISTA DE

CIENCIAS DE LA VIDA

pISSN:1390-3799; eISSN:1390-8596

http:/ / doi.org/10.17163/lgr.n29.2019.03
Artículo científico / Scientific paper

RELLENOS SANITARIOS

\title{
EFICIENCIA Y CONFIABILIDAD DE MODELOS DE ESTIMACIÓN DE BIOGÁS EN RELLENOS SANITARIOS
}

\author{
EFFICIENCY AND RELIABILITY OF THEORETICAL MODELS OF BIOGAS FOR \\ LANDFILLS
}

\section{Pablo Emilio Escamilla García*[0}

Escuela Superior de Comercio y Administración Unidad Tepepan, Instituto Politécnico Nacional, Ciudad de México, México

*Autor para correspondencia: peescamilla@ipn.mx

\section{Resumen}

Este artículo muestra un análisis comparativo de las emisiones de biogás generadas en un relleno sanitario al aplicar el modelo mexicano de biogás, el modelo de la Agencia de Protección Ambiental de los Estados Unidos de América (EPA) y comparar los resultados con datos obtenidos in-situ. Las estimaciones con los modelos teóricos y la medición en campo se realizaron en 36 pozos de venteo de un relleno sanitario ubicado en el Estado de México, México, con una recepción diaria de 3500 kilogramos de RSU. Los resultaron in-situ mostraron una generación de biogás $\left(\mathrm{CH}_{4}, \mathrm{CO}_{2}\right.$ y $\mathrm{O}_{2}$ ) con una frecuencia media de $35,44 \mathrm{~Hz}(1 / \mathrm{s})$ y emisiones de metano de $3355,99 \mathrm{~m}^{3} / \mathrm{hr}$. En contraste los modelos teóricos estimaron valores para el año 2018 de $6270,57 \mathrm{~m}^{3} / \mathrm{hr}$ para el modelo de la EPA y $8379,52 \mathrm{~m}^{3} / \mathrm{hr}$ para el modelo mexicano de biogás. Los resultados mostraron variaciones significativas en las estimaciones de los modelos teóricos versus la medición in-situ. La información generada permite discutir la confiabilidad del uso de modelos teóricos para formular proyectos de aprovechamiento y valorización de RSU al considerar los altos montos de inversión que implican y que las proyecciones de generación de energía se basan en la frecuencia de generación del flujo de biogás estimado en el relleno.

Palabras clave: Biogás, metano, relleno sanitario, estimación teórica.

\begin{abstract}
This paper highlights a comparative analysis of biogas emissions produced in a Mexican landfill. The Mexican biogas model, the model of the Environmental Protection Agency of the United States of America (EPA) were applied in order to compare results with data obtained in-situ. The sanitary landfill located in the State of Mexico, Mexico, has 36 wells with a daily reception of 3500 kilograms of MSW. The results showed an in-situ generation of biogas $\left(\mathrm{CH}_{4}\right.$, $\mathrm{CO}_{2}$ and $\mathrm{O}_{2}$ ) with an average frequency of $35,44 \mathrm{~Hz}(1 / \mathrm{s})$ and methane emissions of $3355,99 \mathrm{~m}^{3} / \mathrm{hr}$. The theoretical models estimated values for the year 2018 of $6270,57 \mathrm{~m}^{3} / \mathrm{hr}$ for the EPA model and 8379,52 $\mathrm{m}^{3} / \mathrm{hr}$ for the Mexican
\end{abstract}


biogas model. The results showed significant variations in the estimates of the theoretical models versus in-situ measurements. This result discusses the reliability of the use of theoretical models to formulate projects for the utilization and valorization of MSW, considering the high amounts of investment involved and that the projections of power generation are based on the frequency of generation of the estimated biogas flow in the landfill.

Keywords: Landfill gas, methane, landfill, theoretical estimation.

Forma sugerida de citar: Escamilla García Pablo E. (2019). Eficiencia y confiabilidad de modelos de estimación de biogás en rellenos sanitarios. La Granja: Revista de Ciencias de la Vida. Vol. 29(1):3244. http://doi.org/10.17163/lgr.n29.2019.03.

IDs Orcid:

Pablo Emilio Escamilla García: http:/ / orcid.org/0000-0001-6704-521X 


\section{Introducción}

El biogás es una mezcla de metano $\left(\mathrm{CH}_{4}\right)(40 \%-$ $70 \%)$, dióxido de carbono $\left(\mathrm{CO}_{2}\right)$ y otros gases (hidrógeno, nitrógeno, oxígeno y sulfuro de hidrógeno) generados por la fermentación de materiales orgánicos (Gautam, Baral y Herat, 2009). Este gas es el resultado de la fermentación de metano de diferentes materiales, como materias primas agrícolas, productos agrícolas, desperdicios de alimentos y heces líquidas o sólidas (Iglinski y col., 2012). La combustión del biogás permite la recuperación de energía y se ha utilizado ampliamente en centrales térmicas y eléctricas, entre otras aplicaciones industriales (Tampio y col., 2014). El biogás se puede usar como fuente de energía y se puede usar en motores combinados de calor y energía. También se puede utilizar como sustituto del gas natural al eliminar el $\mathrm{CO}_{2}$ del $\mathrm{CH}_{4}$. Por tanto, el biogás es un combustible versátil que se puede usar tanto para la generación de energía como para la industria química (Scholz, Melin y Wessling, 2013).

El biogás se genera en grandes volúmenes, principalmente en rellenos sanitarios. En los rellenos sanitarios, la materia orgánica se descompone en ausencia de oxígeno que resulta en la emisión de biogás a la atmósfera (Colling y col., 2016). Los rellenos sanitarios de Residuos Sólidos Urbanos (RSU) son la tercera fuente de emisiones de metano relacionadas con la actividad humana a nivel mundial, esto representa aproximadamente el $15,4 \%$ de estas emisiones (EPA, 2016). Al mismo tiempo, las emisiones de metano de los rellenos sanitarios representan una oportunidad perdida para capturar y utilizar un recurso con potencial energético (Cabrera y Ortiz, 2011). El control y el uso de este gas deben estimar, con una certeza razonable, tanto la producción diaria como la producción acumulada de metano $\left(\mathrm{CH}_{4}\right)$ a largo plazo. Sin embargo, de acuerdo con Calvo y col., (2005), independientemente del método seleccionado para realizar estimaciones, las metodologías deben considerar que: 1) el diagnóstico solo es válido en el momento de la evaluación y su validez disminuye con el tiempo si el relleno sanitario no se monitorea periódicamente; 2) la metodología solo se puede realizar para rellenos sanitarios de RSU independientemente de la escala de recepción; 3) la composición de los desechos del relleno se puede obtener a partir de datos históricos reportados, datos de caracterización de desechos promedios en una población o de la caracterización in situ.
Se han realizado numerosas investigaciones que demuestran que el biogás en los rellenos sanitarios se produce durante largos períodos de tiempo, incluso después de que cesa la disposición de desechos (Pillai, 2018; Lombardi y Carnevale, 2016; Dace y col., 2015; Xiaoli y col., 2011). Sin embargo, la acumulación de dioxinas, furanos y otras emisiones de gases tóxicos en los rellenos crean severos riesgos ambientales y de salud pública en las poblaciones circundantes (Gomez y col., 2018; Kret y col., 2018; Hirata y col., 1995; Bramryd, 1997; Meadows, Franklin y Campbell, 1997). Por lo tanto, el biogás debe ser monitoreado para garantizar un control adecuado de estas emisiones. Este tratamiento normalmente implica la captura y utilización de biogás para fines de producción de energía.

La viabilidad económica de los proyectos para construir y operar tecnologías de aprovechamiento y captura de biogás requiere información precisa sobre la composición del gas y sobre todo sobre las proyecciones estimadas de generación (Chakrabarty, Boksh y Chakrabortye, 2013). La cantidad de biogás producido en los sitios de disposición final varía en función de la cantidad de residuos, el tipo de desecho, el contenido de humedad, la temperatura y las prácticas de manejo, por lo que es necesario realizar una estimación de los gases presentes para cuantificar las emisiones (Knox, 2005). La estimación del metano generado por los RSU se puede realizar utilizando metodologías como el modelo EPA y el modelo mexicano de biogás, que son modelos empíricos basados en una ecuación de primer orden para la degradación de la materia orgánica. Dichas metodologías asumen que la generación de biogás alcanza su máximo después de un período de tiempo anterior a la generación de metano, este período es un año después de la colocación de los residuos sólidos para la generación de biogás; Después de un año de disponer RSU, la generación de biogás disminuye exponencialmente mientras se consume la fracción orgánica de desechos (Urrego y Rodríguez, 2016).

De acuerdo con lo anterior, esta investigación considera la aplicación de dos modelos teóricos (modelo EPA y modelo mexicano) para la estimación de biogás en un relleno sanitario de RSU. Los resultados se comparan con mediciones precisas obtenidas in situ. Esto permite identificar el grado de confiabilidad y eficiencia de los modelos teóricos versus la medición real in situ al comparar variaciones y analizar parámetros y aspectos que pueden causar po- 
sibles inconsistencias.

\section{Materiales y métodos}

La investigación se realizó aplicando diferentes metodologías para estimar el biogás generado en un relleno sanitario en el Estado de México, México. Estas cifras se compararon con mediciones reales obtenidas con un analizador de gases (GA5000) para identificar la efectividad en modelos teóricos.

Tabla 1. Información de alimentación a modelos teóricos.

\begin{tabular}{cc}
\hline Parámetro & Valor \\
\hline Año de apertura & 2010 \\
Año proyectado de clausura & 2037 \\
Inicio de sistema de captura & 2017 \\
Cantidad promedio anual de recepción de residuos & 1105427 \\
Proyección de residuos en el relleno en año de clausura & 29846539 \\
Profundidad del relleno sanitario & $65 \mathrm{~m}$ \\
Superficie en acres & $36(1$ por pozo $)$ \\
Contenido de metano en biogás & $50.00 \%$ \\
Eficiencia de captura & $85.00 \%$ \\
Tamaño del proyecto & Mínimo \\
$\%$ del área con residuos con sistema de captura & 80 \\
\hline
\end{tabular}

Fuente: Encuestas en relleno sanitario.

\subsection{Medición in situ}

El sitio de muestreo fue un relleno sanitario ubicado en una latitud de 19,320539 y una longitud de 98,808288 , con una extensión de $255,619 \mathrm{~m}^{2}$ y ubicado a 2260 metros sobre el nivel del mar con una temperatura promedio de $16,51^{\circ} \mathrm{C}$ y $19,50^{\circ} \mathrm{C}$ y una precipitación pluvial promedio anual de 600 a 800 milímetros. El relleno recibe un promedio diario de 3500 toneladas de residuos provenientes de la Ciudad de México y de ciertos municipios del Estado de México en México.

El relleno cuenta con 36 pozos de venteo, de los cuales 20 se refieren a pozos con sistema de quema, mientras que 16 solo se usan para liberar biogás a la atmósfera. Las mediciones incluyeron datos de los 36 pozos que están en pleno funcionamiento actualmente. La altura promedio de cada pozo se estimó en $3 \mathrm{~m}$ con una longitud total de $65 \mathrm{~m}$. Los pozos están compuestos por columnas con un tubo perforado de polietileno de alta densidad de 6 pulgadas de diámetro. Los tubos están dispuestos a una distancia de 25 metros entre sí, y cada uno cuenta con 4 ranuras en el perímetro dispuestas a lo largo de la longitud del tubo a una distancia de $25 \mathrm{~cm}$ entre ellas.
La medición se realizó en el periodo comprendido entre el 12 al 18 de mayo de 2018 . Se realizaron repeticiones triples en horarios de 9:00 hrs, 14:00 hrs, 18:00 hrs. a fin de considerar diferentes temperaturas ambientes. Se utilizó un analizador portátil de biogás modelo GA5000. Inicialmente se tomaron mediciones de presión barométrica y presión relativa y posteriormente se monitoreo $\mathrm{CH}_{4}$ y $\mathrm{CO}_{2}$ por lapsos de 45 segundos. Los datos fueron analizados mediante el Gas Analyser Manager Software (GAM).

Para las estimaciones con los modelos teóricos se utilizaron los datos de la Tabla 1 para alimentar los algoritmos del Modelo de México de Biogas y el modelo de la Environmental Protection Agency (EPA):

\subsection{Modelo EPA}

El modelo de la EPA requirió datos relacionados con la tasa promedio anual de desechos eliminados, el número de años que el relleno ha estado abierto, el año proyectado de clausura, el potencial de los desechos eliminados para generar metano y la tasa de metano. Para las estimaciones subsecuentes se 
aplicó la siguiente ecuación de primer orden:

$$
L F G=2 \times L_{0} \times R \times\left(e^{-k \times C} e^{-k \times T}\right)
$$

Donde LFG es la cantidad total de biogás generado en el año actual o en consideración $\left(\right.$ pies $\left.^{3}\right)$; $L_{0}$ es el potencial total de generación de metano de los residuos $\left(\right.$ pies $\left.^{3} / l b\right) ; R$ es el promedio anual de residuos dispuestos durante la vida del relleno (libras); $k$ es la tasa anual de generación de metano (1/año); $T$ es número de años de operación del relleno (años); $C$ es el tiempo transcurrido desde el cierre del relleno sanitario (años) (EPA, 2017). El valor de $L o$ y k se estimaron con base en la Tabla 2.

Tabla 2. Parámetros para Lo y K para rellenos sanitarios convencionales.

\begin{tabular}{ll}
\hline Parámetros del modelo & Valor \\
\hline$K$ & 0,050 por año \\
Lo & $170 \mathrm{~m}^{3} /$ ton \\
\hline
\end{tabular}

Fuente: (EPA, 2016).

Tabla 3. Índice de potencial de generación de metano (Lo).

\begin{tabular}{cc}
\hline Precipitación pluvial anual $(\mathrm{mm} / \mathbf{a n ̃ o})$ & $L o\left(\mathrm{~m}^{3} /\right.$ Ton $)$ \\
\hline $0-249$ & 60 \\
$250-499$ & 80 \\
$>500$ & 84 \\
\hline
\end{tabular}

Fuente: Adaptado de Stege y J., (2009).

Tabla 4. Tasa de generación de metano $(K)$.

\begin{tabular}{cc}
\hline Precipitación pluvial anual $(\mathrm{mm} / \mathbf{a n ̃ o})$ & Lo $\left(\mathrm{m}^{3} /\right.$ Ton $)$ \\
\hline $0-249$ & 0,040 \\
$250-499$ & 0,050 \\
$500-999$ & 0,065 \\
$>1000$ & 0,080 \\
\hline
\end{tabular}

Fuente: Adaptado de Stege y J., (2009).

\subsection{Modelo mexicano}

El modelo utilizó la siguiente información para estimar la generación y recuperación de biogás: 1) La cantidad de desechos depositados anualmente en el relleno sanitario, 2) El año de apertura y cierre del sitio, 3) La tasa de generación de metano $(k), 4) \mathrm{La}$ generación potencial de metano $(L o), 5)$ El factor de corrección de metano $(M C F), 6)$ El factor de ajuste por incendios $(F), 7)$ La eficiencia de recuperación del sistema de captura. Para estimar la tasa de generación de biogás para cada año se utilizó la ecuación de degradación de primer grado, como sigue:

$$
Q_{L F G}=\sum_{t=1}^{n} \sum_{j=0,1}^{1} 2 k L o\left[\frac{M i}{10}\right]\left(e^{-k t_{i j}}\right)(M C F)(F)
$$

Donde: $Q_{L F G}=$ Flujo máximo esperado de biogás $\left(\mathrm{m}^{3}\right) ; \mathrm{i}=$ aumento de tiempo de 1 año; $\mathbf{n}=$ (año de cálculo) - (año inicial de eliminación de desechos); $\mathrm{j}=$ Incremento de tiempo en 0,1 años; $\mathrm{k}$ = Generación de metano (1 / año); Lo = Generación potencial de metano $\left(\mathrm{m}^{3} / \mathrm{Mg}\right) ; \mathrm{Mi}=$ Masa de residuos dispuestos en el año i (Mg); $t_{i j}=$ Edad de la sección j de la masa de desechos Mi dispuesta en el año i (años decimales); MCF = factor de corrección de metano; $\mathrm{F}=$ Factor de ajuste por incendios.

La ecuación anterior estimó la generación de biogás utilizando las cantidades de residuos eliminados acumulados durante un año. Las proyecciones para varios años se desarrollan variando la proyección anual y luego iterando la ecuación. La genera- 
ción total de biogás es igual al doble de la generación de metano calculada. La composición de biogás asumida en el modelo fue de $50 \%\left(\mathrm{CH}_{4}\right)$ y $50 \%$, que incluyen dióxido de carbono $\left(\mathrm{CO}_{2}\right)$ y otros compuestos. La función de degradación exponencial supone que la generación de biogás se encuentra en su máximo un período antes de la generación de metano. El modelo asumió un período de seis meses entre la colocación de los residuos y la generación de biogás. Para cada unidad de residuos, después de seis meses, se asumió que la generación de biogás disminuye exponencialmente a medida que se consume la fracción orgánica de los desechos. El año máximo de generación usualmente ocurre en el año de cierre o el año siguiente (dependiendo del índice de disposición en los años finales).
Para el cálculo de la tasa de generación de metano y del índice de potencial de metano se utilizaron los siguientes parámetros (Tablas 3 y 4).

\section{Resultados y discusión}

\subsection{Resultados de medición in situ}

En la Tabla 12 se muestran los resultados obtenidos luego de muestrear 36 pozos en el relleno sanitario. La concentración de metano, dióxido de carbono, oxígeno, así como la frecuencia de generación mostró valores similares para cada pozo. Los valores promedio para el relleno sanitario se presentan en la Tabla 5.

Tabla 5. Valores promedios obtenidos in-situ para el relleno sanitario.

\begin{tabular}{ccccc}
\hline $\mathrm{CH}_{4}(\mathbf{\%})$ & $\mathrm{CO}_{2}(\boldsymbol{\%})$ & $\mathrm{O}_{2}(\boldsymbol{\%})$ & $\mathbf{H z}(\mathbf{1} / \mathbf{s})$ & $\mathrm{CH}_{4}$ por hora \\
\hline 50.29 & 46.88 & 1.01 & 35.44 & 3355.99 \\
\hline
\end{tabular}

\subsection{Resultados del modelo mexicano de biogás}

La estimación se realizó al aplicar la ecuación [1] de degradación de primer orden. Los datos utilizados para alimentar el modelo se pueden ver en la Tabla 1. El modelo proporcionó valores para el índice de generación de metano $(k)$ y la generación poten- cial de metano $(L o)$ que fueron verificados por los valores propuestos por Aguilar, Taboada y Ojeda, (2011). Estos valores se desarrollaron utilizando datos climáticos, caracterización de residuos y prácticas de eliminación precargados en los modelos teóricos. La Tabla 6 muestra los parámetros utilizados para el modelado.

Tabla 6. Parámetros para modelaje (Modelo mexicano de biogás).

\begin{tabular}{|c|c|c|c|c|}
\hline \multicolumn{5}{|c|}{ Contenido de metano en biogás: $50 \%$} \\
\hline \multicolumn{5}{|c|}{ Factor de corrección de metano (MCF): 1.0} \\
\hline Categoría de residuos & $\begin{array}{l}\text { Degradación } \\
\text { rápida }\end{array}$ & $\begin{array}{l}\text { Degradación } \\
\text { moderadamente } \\
\text { rápida }\end{array}$ & $\begin{array}{c}\text { Degradación } \\
\text { moderadamente } \\
\text { lenta }\end{array}$ & $\begin{array}{l}\text { Degradación } \\
\text { lenta }\end{array}$ \\
\hline $\begin{array}{l}\mathrm{CH}_{4}(k) \text { índice de } \\
\text { generación: }\end{array}$ & 0.16 & 0.075 & 0.032 & 0.016 \\
\hline $\begin{array}{l}\mathrm{CH}_{4}(\mathrm{Lo}) \text { potencial de } \\
\text { generación }\left(\mathrm{m}^{3} / \mathrm{Mg}\right) \text { : }\end{array}$ & 69 & 138 & 214 & 202 \\
\hline
\end{tabular}

La Tabla 7 presenta los valores obtenidos después del modelaje. Cabe señalar que los modelos teóricos (EPA y modelo mexicano) estiman la generación en función de la caracterización pre establecida. Asimismo, el modelo estima la acumulación de residuos aumentando la cantidad de residuos dispuestos por año. Aunque la Tabla 7 presenta datos hasta 2025, el modelo resultó en valores hasta 2037, año proyectado para el cierre del relleno sanitario. 
Tabla 7. Proyecciones de generación y recuperación de biogás en modelo mexicano.

\begin{tabular}{|c|c|c|c|c|c|c|c|c|}
\hline \multirow{2}{*}{ Año } & \multirow{2}{*}{$\begin{array}{c}\text { Disposición } \\
\text { (Mg/año) }\end{array}$} & \multirow{2}{*}{$\begin{array}{c}\text { Disposición } \\
\text { acumulada (Mg) }\end{array}$} & \multicolumn{3}{|c|}{ Generación de biogás } & \multicolumn{3}{|c|}{ Recuperación estimada de biogás } \\
\hline & & & $\left(m^{3} / h r\right)$ & $\left(\right.$ pies $\left.^{3} / \mathrm{min}\right)$ & ( $\mathrm{mm}$ Btu/hr) & $\left(m^{3} / h r\right)$ & $\left(\right.$ pies $\left.^{3} / \mathrm{min}\right)$ & ( $\mathrm{mm}$ Btu/hr) \\
\hline 2010 & 981600 & 981600 & 0 & 0 & 0 & 0 & 0 & 0 \\
\hline 2011 & 1001200 & 1982800 & 1424 & 838 & 25.4 & 0 & 0 & 0 \\
\hline 2012 & 1021200 & 3004000 & 2706 & 1593 & 48.4 & 0 & 0 & 0 \\
\hline 2013 & 1041600 & 4045600 & 3866 & 2275 & 69.1 & 0 & 0 & 0 \\
\hline 2014 & 1062400 & 5108000 & 4922 & 2897 & 88 & 0 & 0 & 0 \\
\hline 2015 & 1083600 & 6191600 & 5889 & 3466 & 105.2 & 0 & 0 & 0 \\
\hline 2016 & 1105427 & 7297027 & 6781 & 3991 & 121.2 & 0 & 0 & 0 \\
\hline 2017 & 1127500 & 8424527 & 7608 & 4478 & 135.9 & 4032 & 2373 & 72 \\
\hline 2018 & 1150100 & 9574627 & 8380 & 4932 & 149.7 & 4441 & 2614 & 79.4 \\
\hline 2019 & 1173100 & 10747727 & 9105 & 5359 & 162.7 & 4825 & 2840 & 86.2 \\
\hline 2020 & 1196600 & 11944327 & 9790 & 5762 & 174.9 & 5189 & 3054 & 92.7 \\
\hline 2021 & 1220500 & 13164827 & 10442 & 6146 & 186.6 & 5534 & 3257 & 98.9 \\
\hline 2022 & 1244900 & 14409727 & 11065 & 6513 & 197.7 & 5865 & 3452 & 104.8 \\
\hline 2023 & 1269800 & 15679527 & 11665 & 6866 & 208.4 & 6182 & 3639 & 110.5 \\
\hline 2024 & 1295200 & 16974727 & 12244 & 7207 & 218.8 & 6489 & 3819 & 116 \\
\hline 2025 & 1321100 & 18295827 & 12807 & 7538 & 228.8 & 6788 & 3995 & 121.3 \\
\hline
\end{tabular}

\subsection{Resultados del modelo EPA}

El modelo EPA utiliza una herramienta desarrollada para el Landfill Methane Outreach Program
(LMOP) para estimar emisiones y costos en proyectos de captura y aprovechamiento de biogás (Tabla 8). En la Tabla 9 se muestran los principales valores obtenidos en el modelo EPA.

Tabla 8. Generación, recolección y utilización de biogás.

\begin{tabular}{|c|c|}
\hline \multicolumn{2}{|l|}{ Parámetros para modelación: } \\
\hline $\begin{array}{l}\text { Tasa de generación de metano, k (1/año): } \\
\end{array}$ & 0.04 \\
\hline Capacidad de generación de metano, LO ( pies $^{3} /$ ton $)$ : & 3204 \\
\hline Contenido de metano en LFG: & $50.00 \%$ \\
\hline \multicolumn{2}{|c|}{ Generación estimada durante operación del relleno $\left(\right.$ pies $^{3} / \mathrm{min}$ LFG): } \\
\hline Mínimo: & 3291 \\
\hline Promedio anual & 5663 \\
\hline Máximo & 7659 \\
\hline \multicolumn{2}{|l|}{ Recuperación durante operación del relleno $\left(\mathrm{pies}^{3} / \mathrm{min}\right.$ LFG): } \\
\hline $\begin{array}{c}\text { Mínimo: } \\
\end{array}$ & 2798 \\
\hline Promedio anual: & 4814 \\
\hline Máximo: & 6510 \\
\hline Tamaño del proyecto: & Mínimo \\
\hline Tasa de generación $\left(\right.$ pies $^{3} / \min$ LFG): & 2798 \\
\hline \multicolumn{2}{|l|}{ Utilizado por el poyecto: $\left(\right.$ pies $^{3} / \mathrm{min}$ LFG): } \\
\hline $\begin{array}{l}\text { Promedio anual } \\
\end{array}$ & 2601.8 \\
\hline Eficiencia de recuperación de biogás: & $85.00 \%$ \\
\hline
\end{tabular}

\section{Discusión}

Los valores obtenidos mostraron diferencias significativas en los niveles de biogás. Los modelos teó- ricos (EPA y modelo mexicano) estimaron valores que resultan de modelar datos en ecuaciones de degradación de primer orden. Los resultados se muestran en la Figura 1: 
Tabla 9. Proyecciones de generación de metano $\left(\mathrm{m}^{3} / \mathrm{hr}\right)$ en modelo EPA. Valores obtenido en modelación.

\begin{tabular}{cc}
\hline Año & $m^{3} / h r$ \\
\hline 2010 & 0 \\
2011 & 897.83 \\
2012 & 1760.46 \\
2013 & 2589.27 \\
2014 & 3385.57 \\
2015 & 4150.66 \\
2016 & 4885.74 \\
2017 & 5592 \\
2018 & 6270.57 \\
2019 & 6922.53 \\
2020 & 7548.93 \\
2021 & 8150.76 \\
2022 & 8729 \\
2023 & 9284.56 \\
2024 & 9818.34 \\
2025 & 10331.19 \\
\hline
\end{tabular}

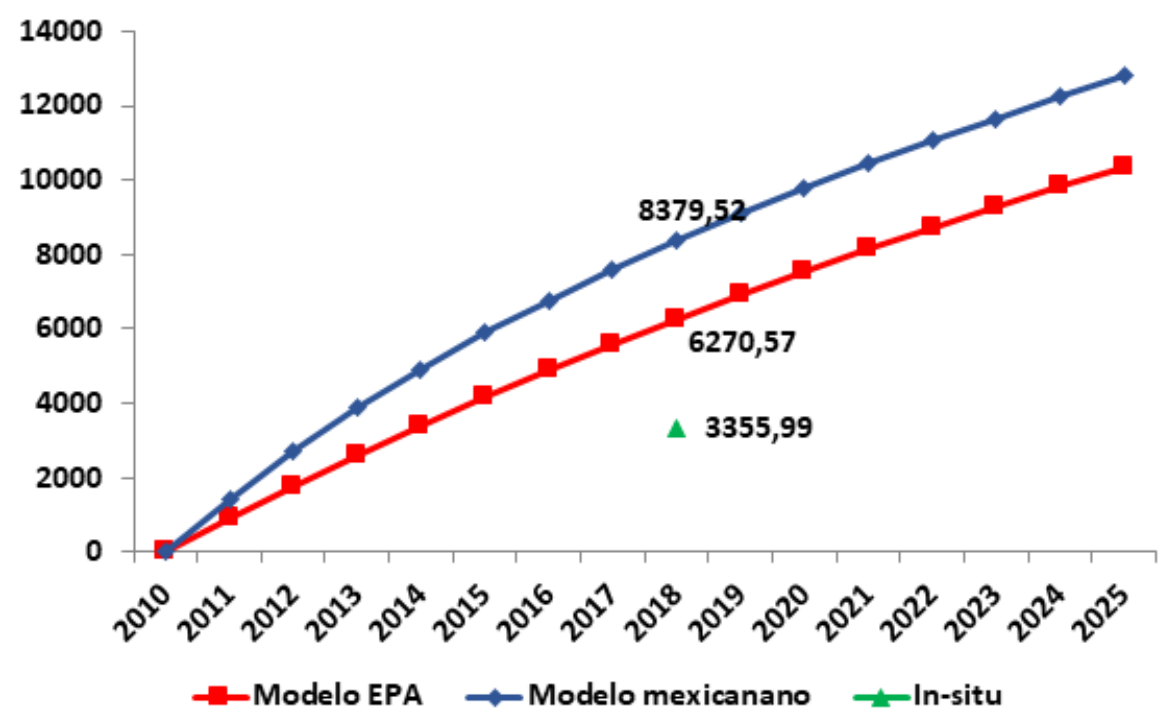

Figura 1. Generación estimada de metano $\left(\mathrm{m}^{3} / \mathrm{hr}\right)$.

La Figura 1 muestra las emisiones de metano en metros cúbicos por hora. Es posible apreciar que los modelos teóricos estimaron valores para el 2018 de $8379,52 \mathrm{~m}^{3} / \mathrm{hr}$ (modelo mexicano) y $6270,57 \mathrm{~m}^{3} / \mathrm{hr}$ (Modelo EPA). Estos valores contrastan significativamente con el valor real in situ que muestra que en el año 2018 la generación es de 3355,99 $\mathrm{m}^{3} / \mathrm{hr}$. Las variaciones en los resultados obedecen a diferentes elementos, en primera estancia están los supuestos de los modelos teóricos.

En el modelo de la EPA, la estimación de la generación de biogás (LFG) producida se multiplica por la eficiencia de recolección para estimar el volumen de final de metano que se puede recuperar. Sin embargo, las proyecciones se calculan con base en estimaciones de eficiencia de captura razonables para rellenos sanitarios que cumplen con los estándares establecidos en Titulo 40, parte 258 del 
Código de Regulaciones Federales en los Estados Unidos de América. Las eficiencias de recolección que reportan estos rellenos sanitarios oscilan entre el $50 \%$ y el $95 \%$ de eficiencia, por lo que el modelo asume un $75 \%$ de eficiencia de captura. Adicionalmente, el modelo de la EPA asume instalaciones con un sistema integral de recolección y tratamiento que aumentará su eficiencia y los años proyectados. En consecuencia, la variación del valor real in situ con las estimaciones del modelo EPA es entendible ya que los rellenos sanitarios en México carecen de sistemas integrales que garanticen una eficiencia en la captura de biogás, y sobre todo que los rellenos sanitarios en México en su gran mayoría basan su operación en métodos rudimentarios y tecnologías obsoletas (Escamilla y col., 2016).

Los resultados obtenidos en el modelo mexicano de biogás, presentan una variación aun mayor que los datos del modelo de la EPA. La diferencia de los $\mathrm{m}^{3}$ por hora de metano generado en 2018 entre la medición real in situ y el modelo EPA fue de 2914,58 $\mathrm{m}^{3} / \mathrm{hr}$, mientras que la diferencia con el modelo mexicano de biogás fue de $5023,53 \mathrm{~m}^{3} / \mathrm{hr}$. Esto implica una diferencia 2.5 veces mayor que la emisión real. El modelo mexicano asigna automáticamente los valores de $\mathrm{k}$ y Lo de acuerdo a los valores de la Tabla 10.

Tabla 10. Valores del Índice de Generación de Metano $(k)$ y de Generación Potencial de Metano (Lo) en modelo Mexicano de biogás por región.

\begin{tabular}{ccccccccccc}
\hline $\begin{array}{c}\text { Categoría } \\
\text { de } \\
\text { Residuos }\end{array}$ & Región1 & Región 2 & \multicolumn{2}{c}{ Región3 } & Región 4 & \multicolumn{2}{c}{\begin{tabular}{c} 
Región 5 \\
\cline { 2 - 12 }
\end{tabular}} & Sureste & Oeste & \multicolumn{4}{c}{$\begin{array}{c}\text { Centro/ } \\
\text { Interior* }\end{array}$} & Noreste & \multicolumn{2}{c}{$\begin{array}{c}\text { Noroeste \& } \\
\text { Interior } \\
\text { Norte }\end{array}$} \\
\cline { 2 - 12 } & $k$ & $L o$ & $k$ & $L o$ & $k$ & $L o$ & $k$ & $L o$ & $k$ & $L o$ \\
\hline $\mathbf{1}$ & 0.3 & 69 & 0.22 & 69 & 0.16 & 69 & 0.15 & 69 & 0.1 & 69 \\
\hline $\mathbf{2}$ & 0.13 & 115 & 0.1 & 126 & 0.075 & 138 & 0.07 & 138 & 0.05 & 149 \\
\hline $\mathbf{3}$ & 0.05 & 214 & 0.04 & 214 & 0.032 & 214 & 0.03 & 214 & 0.02 & 214 \\
\hline $\mathbf{4}$ & 0.025 & 202 & 0.02 & 202 & 0.016 & 202 & 0.015 & 202 & 0.01 & 202 \\
\hline
\end{tabular}

Fuente: Adaptado de (Stege y J., 2009).

Como se puede observar en la Tabla 10, el índice de generación de metano usado en la estimación se asigna en función de la localización del relleno sanitario a evaluar. El modelo establece cinco regiones geográficas. Cada región identifica primeramente la precipitación pluvial y la temperatura promedio de la zona. Posteriormente la categoría de residuos se refiere a 1) Sitio sin manejo; 2) Sitio con manejo; 3 ) Sitio semi-aeróbico y 4) Condición desconocida. Si no existe información precisa sobre la caracterización de los residuos, el modelo asume valores de caracterización por cada zona.

El considerar el hecho que el modelo mexicano estima los valores con base en información particular de México, debería brindar una mayor confiabilidad en los resultados. Estos resultados tendrían que estar en niveles con una variación aceptable en relación con los datos de mediciones reales in situ. Sin embargo, como se evidenció, las estimaciones mostraron una variabilidad significativa. El modelo de la EPA reportó valores aún más cercanos a los datos reales in situ. Esto es una situación importante ya que se demuestra que el modelo mexicano, el cual dadas sus características tendría que estimar valores cercanos a la realidad, mostró lo contrario al reportar los valores más alejados de la medición real. El principal punto débil del modelo mexicano y que podría explicar la variación tan amplia en las estimaciones es la información sobre la caracterización de los residuos, particularmente las fracciones orgánicas. La información estadística relacionada con la caracterización de residuos en México es escasa y presenta niveles bajos de confiabilidad. En un sistema integral de gestión de RSU la caracterización de los residuos es fundamental no solo para establecer estimaciones de metano en la fracción orgánica sino para establecer estrategias para migrar a sistemas de cero residuos (Ayeleru, Okonta y F., 2018; Adeniran, Nubi y Adelopo, 2017).

El flujo de residuos en un relleno sanitario y su ca- 
racterización varía en función factores de cada región como: actividades económicas, clima, cultura, energía, fuentes de generación, entre otras. Los países en vías de desarrollo tienden a generar una proporción significativa de residuos orgánicos, mien- tras que los países desarrollados presentan proporciones mayores en la fracción inorgánica (Chang y col., 2011). La Tabla 11 ilustra los diferentes valores promedio de composición de acuerdo con el tipo de economía.

Tabla 11. Composición de residuos por nivel economico*. Elaborado a partir de datos de Banco Mundial2012

\begin{tabular}{lcccccc}
\hline Nivel de ingreso & $\begin{array}{c}\text { Orgánico } \\
(\boldsymbol{\%})\end{array}$ & $\begin{array}{c}\text { Papel } \\
(\boldsymbol{\%})\end{array}$ & $\begin{array}{c}\text { Plástico } \\
(\mathbf{\%})\end{array}$ & $\begin{array}{c}\text { Vidrio } \\
(\boldsymbol{\%})\end{array}$ & $\begin{array}{c}\text { Metal } \\
(\boldsymbol{\%})\end{array}$ & $\begin{array}{c}\text { Otros } \\
(\boldsymbol{\%})\end{array}$ \\
\hline Ingreso bajo & 64 & 5 & 8 & 3 & 3 & 17 \\
Ingreso medio bajo & 59 & 9 & 12 & 3 & 2 & 15 \\
Ingreso medio alto & 54 & 14 & 11 & 5 & 3 & 13 \\
Ingreso alto & 28 & 31 & 11 & 7 & 6 & 17 \\
\hline
\end{tabular}

*Nota: La Tabla fue construida con información del Banco Mundial, que incluye datos de 105 países clasificados por ingreso y con tasas de generación de RSU en el periodo 2006 a 2012. La tasa de generación incluyó áreas urbanas solamente y en algunos países los valores de composición fueron de una sola ciudad.

La Tabla 11 muestra que los países de bajos ingresos tienen una fracción orgánica del $64 \%$ en comparación con el $28 \%$ en países de alto ingreso. Esto demuestra que, a medida que un país incrementa sus niveles de desarrollo económico, se tiene una repercusión en el flujo de RSU y la fracción orgánica disminuye. En consecuencia, las estimaciones del modelo mexicano de biogás presentan una baja confiablidad debido a los datos de caracterización de residuos que utiliza como base. El modelo asume una alta concentración de fracción orgánica mientras que los datos reales demuestran que este componente es menor al estimado. Los valores reportados por los modelos teóricos en esta investigación tienen un comportamiento similar, esto es debido al modelo matemático que aplican y a la degradación exponencial del residuo estimada. La variabilidad significativa entre los datos de los modelos teóricos y la medición in situ que se reporta en este artículo se alinean con los resultados reportados por Urrego y Rodríguez, (2016) quienes encontraron variaciones atípicas entre los modelos teóricos y un modelo del Grupo Intergubernamental de Expertos sobre el Cambio Climático (IPCC).

Sin embargo, esta investigación se ubica en México, se esperaba que el modelo mexicano brindará información aproximada pero confiable de generación de metano. Investigaciones han demostrado el impacto negativo que una incorrecta cuantificación de biogás en un relleno sanitario puede tener en pro- yectos de generación de energía (Judy y col., 2018; Blanco y col., 2018; Li y col., 2018). En consecuencia, los modelos teóricos, particularmente el modelo mexicano de biogás demostró ser poco confiable para generar información preliminar sobre emisiones de metano.

En México la provisión de información sobre caracterización de residuos es poco confiable. Se demuestra así que, si bien los modelos teóricos pueden ser una herramienta de uso práctico, los resultados no pueden ser utilizados para definir estrategias y planes de acción sobre todo en proyectos de inversión para generación de energía. La eficiencia y rentabilidad de una planta de valorización de RSU para generación se basa en la frecuencia adecuada y niveles de emisión constante de metano por hora. Es importante que el modelo mexicano de biogás sea actualizado en términos de caracterización de residuos para evitar así la variabilidad en las estimaciones.

\section{Conclusiones}

La medición in situ evidenció emisiones de metano significativamente menores a los valores estimados por los modelos teóricos (in situ $=3355,99 \mathrm{~m}^{3} / \mathrm{hr}$, modelo EPA $=6270,57 \mathrm{~m}^{3} / \mathrm{hr}$, modelo mexicano = $8379,52 \mathrm{~m}^{3} / \mathrm{hr}$ ). Las variaciones en los valores obtenidos se deben a los parámetros que cada modelo asume que discrepan ampliamente de las características reales de los rellenos sanitarios en México. 
El modelo EPA y el modelo mexicano no presentan una variación tan amplia entre si debido al método matemático que aplican (ecuación de primer orden).

La medición in situ mostró que la complejidad de los elementos necesarios para la estimación del biogás generado puede tener un impacto significativo en los resultados. Los modelos teóricos proporcionan proyecciones que pueden utilizarse como información preliminar. Sin embargo, se demuestra que no son confiables y es fundamental realizar una medición con equipo especializado in situ para obtener información útil para la toma de decisiones. Un mo-

\section{Referencias}

Adeniran, A., A. Nubi y A. Adelopo (2017). «Solid waste generation and characterization in the University of Lagos for a sustainable waste management.» En: Journal of the Air and Waste Management Association 67, 3-10. Online: https: / / bit.ly/2Vw2DLl.

Aguilar, Q., P. Taboada y S. Ojeda (2011). «Modelo mexicano para la estimación de la generación de biogás.» En: Revista Académica de la Facultad de Ingeniería, Universidad Autónoma de Yucatán 15.1, págs. 37-45.

Ayeleru, O., F. Okonta y Ntuli F. (2018). «Municipal solid waste generation and characterization in the City of Johannesburg: A pathway for the implementation of zero waste.» En: Waste Management 79, 87-9. Online: https: / / bit.ly/2U6p09E.

Blanco, H. y col. (2018). «Potential of Power-toMethane in the EU energy transition to a low carbon system using cost optimization.» En: $A p$ plied Energy 232.15, 323-340. Online: https://bit. ly/2XumGv9.

Bramryd, T. (1997). «Landfilling in the perspective of the global CO2 balance». En: ed. por Sixth International Landfill Symposium. Sardinia, Italia.

Cabrera, R. y A. Ortiz (2011). Estudio experimental a escala piloto y modelización de la producción de biogás en relleno sanitario. Inf. téc. Centro Interdisciplinario de Investigaciones y Estudios sobre Medio Ambiente y Desarrollo del Instituto Politécnico Nacional.

Calvo, F. y col. (2005). «Environmental diagnosis methodology for municipal waste landfills.» En: Waste Management 25.8, 768-779. Online: https: //bit.ly/2TdByz1. delo teórico puede subestimar o sobreestimar la generación de biogás proyectada. Esto es crítico si dicha información es la base para la implementación de proyectos de generación de energía a partir del biogás. Si los interesados no están en condiciones de realizar una medición in situ, se debe prestar especial atención al modelo teórico elegido para las proyecciones y procurar la provisión certera de información sobre la caracterización de los residuos en el relleno en cuestión, ya que son estos datos los que pueden provocar la variabilidad de los resultados finales.

Chakrabarty, S., M. Boksh y A. Chakrabortye (2013). «Economic viability of biogas and green selfemployment opportunities». En: Renewable and Sustainable Energy Reviews 28, 757-766. Online: https://bit.ly/2EnYLEW.

Chang, S. y col. (2011). "Comprehensive utilization of biogas in Inner Mongolia, China.» En: Renewable and Sustainable Energy Reviews 15.3, 1442-1453. Online: https://bit.ly/2tJfttM.

Colling, A. y col. (2016). «Brazilian recycling potential: Energy consumption and Green House Gases reduction.» En: Renewable and Sustainable Energy Reviews 59, 544-549. Online: https: / / bit. ly/2VrdDJO.

Dace, E. y col. (2015). «Optimization of Landfill Gas Use in Municipal Solid Waste Landfills in Latvia». En: Energy Proceia 72, 293-299. Online: https:/ / bit.ly/2T1sZbb.

EPA (2016). Air Emissions from Municipal Solid Waste Landfills Background Information for Proposed Standards and Guidelines. Inf. téc. EPA-Environmental Protection Agency, Office of Air Quality Planning and Standards. Online: https: / / bit.ly / 2Tlw84S.

- (2017). LFG Energy Project Development Handbook. Landfill Methane Outreach Program. Inf. téc. EPA Environmental Protection Agency. Online: https://bit.ly/2fTvZh9.

Escamilla, P.E. y col. (2016). «Economic feasibility analysis for electrical generation from biogas in waste disposal sites in Mexico City». En: Applied Economics 48 48.59, págs. 5761-5771.

Gautam, R., S. Baral y S. Herat (2009). «Biogas as a sustainable energy source in Nepal: Present status and future challenges.» En: Renewable and 
Sustainable Energy Reviews 13.1, 248-252. Online: https://bit.ly/2BTSj8w.

Gomez, S. y col. (2018). «Dioxins and furans legacy of lindane manufacture in Sabiñánigo (Spain). The Bailín landfill site case study». En: Science of The Total Environment 624, 955-962. Online: https: / / bit.ly/2GPnFRA.

Hirata, T. y col. (1995). «Construction of facilities on closed landfills.» En: ed. por Fifth International Landfill Symposium. Sardinia, Italia.

Iglinski, B. y col. (2012). «Agricultural biogas plants in Poland: Investment process, economical and environmental aspects, biogas potential». En: Renewable and Sustainable Energy Reviews 16.7, 4890â"4900. Online: https:/ / bit.ly/2Eis9fS.

Judy, J. y col. (2018). «Energy balance and diurnal variation in methane production as affected by feeding frequency in Jersey cows in late lactation.» En: Journal of Dairy Science. Online: https: //bit.ly/2EgNruf.

Knox, A. (2005). An Overview of Incineration and EFW Technology as Applied to the Management of $\mathrm{Mu}$ nicipal Solid Waste (MSW). Inf. téc. Energy Subcommittee of the Ontario Environment Industry Association. Online: https:/ / bit.ly/2Tg8hUj.

Kret, J. y col. (2018). «A respiratory health survey of a subsurface smoldering landfill». En: Environmental Research 166, 427-436. Online: https://bit. ly/2tIUx5U.

Li, R. y col. (2018). «Improved methane production and energy recovery of post-hydrothermal liquefaction waste water via integration of zeolite adsorption and anaerobic digestion». En: Science of The Total Environment 651.1, 61-69. Online: https://bit.ly/2TqHgO8.
Lombardi, L. y E.A. Carnevale (2016). «Analysis of an innovative process for landfill gas quality improvement.» En: Energy 109, 1107-1117. Online: https:/ / bit.ly/2H53kad.

Meadows, C., D. Franklin y P. Campbell (1997). «Global methane emissions from solid waste disposal sites». En: ed. por Sixth International Landfill Symposium. Sardinia, Italia.

Pillai J. andRiverol, C. (2018). «Estimation of gas emission and derived electrical power generation from landfills». En: Trinidad and Tobago as study case. Sustainable Energy Technologies and Assessments 29, 139-146. Online: https: / / bit.ly / 2H5xVVe.

Scholz, M., T. Melin y M. Wessling (2013). «Transforming biogas into bio-methane using membrane technology.» En: Renewable and Sustainable Energy Reviews 17, 199â"212. Online: https: / / bit.ly/2BYqcVs.

Stege, G. y Davila J. (2009). Manual del Usuario Modelo Mexicano de Biogas Version 2.0. Online: https: //bit.ly/2GNMIEB.

Tampio, E y col. (2014). "Anaerobic digestion of autoclaved and untreated food waste.» En: Waste Management 34.2, 370-377. Online: https: / / bit. ly/2EjR4ji.

Urrego, E. y J.P. Rodríguez (2016). «Application of methodologies EPA, Mexican and IPCC for estimating biogas, a case study of the sanitary landfill Dona Juana, Bogotá- Colombia». En: Revista Universidad y Salud 18.2, págs. 338-344.

Xiaoli, C. y col. (2011). "Characteristics of vegetation and its relationship with landfill gas in closed landfil». En: Biomass and Bioenergy 35.3, 1295-1301. Online: https://bit.ly/2EjR4ji. 
Tabla 12. Valores obtenidos in situ por pozo de venteo.

\begin{tabular}{|c|c|c|c|c|c|}
\hline Pozo & $\begin{array}{c}\text { Metano } \\
\left(\mathrm{CH}_{4}\right)(\%)\end{array}$ & $\begin{array}{c}\text { Dióxido de } \\
\text { carbono }\left(\mathrm{CO}_{2}\right)(\%)\end{array}$ & $\begin{array}{l}\text { Oxígeno } \\
\left(\mathrm{O}_{2}\right)(\%)\end{array}$ & $\begin{array}{c}\text { Frecuencia } \\
\text { Hz }(1 / s)\end{array}$ & $\begin{array}{c}\text { Emisiones } \\
\text { por hora de } \\
\text { metano }\end{array}$ \\
\hline 1 & 48.8 & 51 & 0.2 & 5.42 & 53.1005715 \\
\hline 2 & 50 & 50 & 0 & 5.06 & 1310.77465 \\
\hline 3 & 49.5 & 50.5 & 0 & 77.57 & 19893.2849 \\
\hline 4 & 49.7 & 50.3 & 0 & 113.77 & 1135.1787 \\
\hline 5 & 51.7 & 48.3 & 0 & 90.1 & 24133.6408 \\
\hline 6 & 49.8 & 50.2 & 0 & 0.3 & 77.4030562 \\
\hline 7 & 38.5 & 59.2 & 2.3 & 0.3 & 59.8397121 \\
\hline 8 & 51.3 & 48.6 & 0 & 76.57 & 20350.8955 \\
\hline 9 & 50.4 & 49.2 & 0.4 & 96.93 & 25310.2398 \\
\hline 10 & 50.4 & 49.6 & 0 & 79.6 & 20785.052 \\
\hline 11 & 51.4 & 48.2 & 0.4 & 3.48 & 35.9105825 \\
\hline 12 & 51.9 & 48.1 & 0 & 2.58 & 142.729123 \\
\hline 13 & 50.7 & 48.9 & 0.3 & 164.1 & 1670.30825 \\
\hline 14 & 51.3 & 48.7 & 0 & 4.02 & 41.4022082 \\
\hline 15 & 50.5 & 49.4 & 0.1 & 130.97 & 1327.83249 \\
\hline 16 & 50.6 & 48.5 & 0.8 & 2.32 & 23.5677753 \\
\hline 17 & 51.4 & 48.6 & 0 & 165.1 & 1703.68884 \\
\hline 18 & 52 & 48 & 0 & 72.13 & 753.007605 \\
\hline 19 & 50.8 & 49.1 & 0.1 & 20.4 & 208.052992 \\
\hline 20 & 50.2 & 49.7 & 0.1 & 0.87 & 8.76805078 \\
\hline 21 & 50.7 & 49.1 & 0.2 & 0.3 & 3.05357999 \\
\hline 22 & 49.9 & 50.1 & 0 & 22.79 & 228.310012 \\
\hline 23 & 53.3 & 46.4 & 0.3 & 0.57 & 6.09933028 \\
\hline 24 & 52.6 & 46.6 & 0.8 & 0.3 & 81.7550352 \\
\hline 25 & 44.6 & 32.9 & 5.3 & 0.3 & 2.68618673 \\
\hline 26 & 54.1 & 45.6 & 0.3 & 4.88 & 53.0025999 \\
\hline 27 & 55.4 & 40.5 & 2.3 & 0.3 & 3.33665348 \\
\hline 28 & 52.7 & 38.3 & 3.1 & 0.3 & 3.17403679 \\
\hline 29 & 54.3 & 44.9 & 0.8 & 4.68 & 51.0182749 \\
\hline 30 & 26 & 18.3 & 12.5 & 0.3 & 1.56593845 \\
\hline 31 & 56.4 & 40.2 & 1.8 & 0.3 & 3.39688188 \\
\hline 32 & 52.9 & 47.1 & 0 & 62.2 & 660.581099 \\
\hline 33 & 49.8 & 50.2 & 0.1 & 15.38 & 153.767929 \\
\hline 34 & 52.8 & 47.2 & 0.1 & 17.3 & 183.383439 \\
\hline 35 & 52.6 & 47.4 & 3.9 & 5.29 & 55.862646 \\
\hline 36 & 51.4 & 48.6 & 0 & 29.07 & 299.977193 \\
\hline
\end{tabular}

Elaborada a partir de mediciones in situ. 